\title{
Failure-experiment-supported optimization of poorly reproducible synthetic conditions for novel lanthanide metal-organic frameworks with two-dimensional secondary building units
}

\author{
Yu Kitamura, ${ }^{[\mathrm{a}]}$ Emi Terado, ${ }^{[\mathrm{a}]}$ Zechen Zhang, ${ }^{[\mathrm{b}]}$ Hirofumi Yoshikawa, ${ }^{[\mathrm{b}]}$ Tomoko Inose, ${ }^{[\mathrm{c}, \mathrm{d}]}$ Hiroshi Uji- \\ i, ${ }^{[c, e]}$ Masaharu Tanimizu, ${ }^{[f]}$ Akihiro Inokuchi, ${ }^{[g]}$ Yoshinobu Kamakura, ${ }^{[a]}$ and Daisuke Tanaka*[a,h]
}

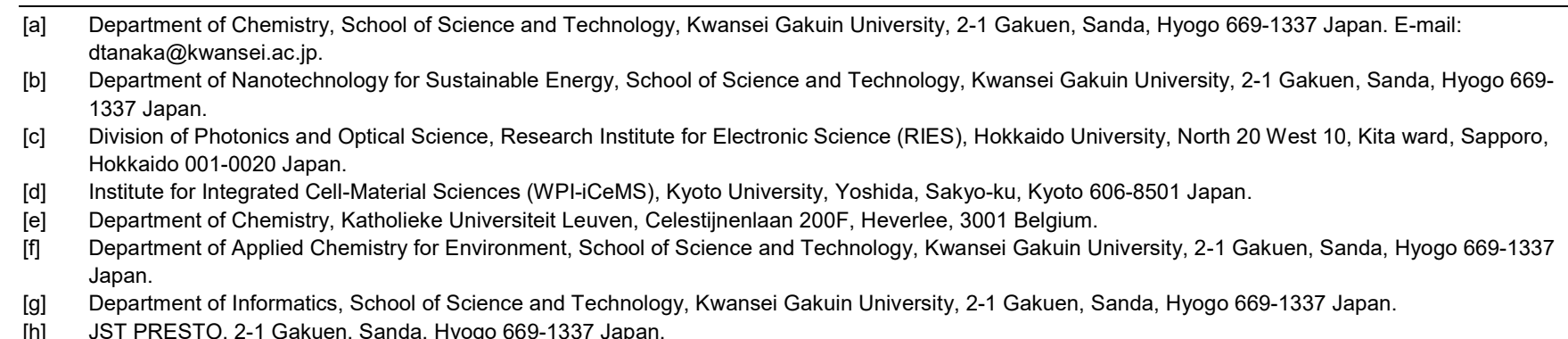

Supporting information for this article is given via a link at the end of the document.

\begin{abstract}
Novel metal-organic frameworks containing lanthanide double-layer-based secondary building units (KGF-3) were synthesized using machine learning (ML). Isolating pure KGF-3 was challenging, and the synthesis was not reproducible because impurity phases were frequently obtained under the same synthetic conditions. Thus, dominant factors for the synthesis of KGF-3 were identified, and its synthetic conditions were optimized using two ML techniques. Cluster analysis was used to classify the obtained PXRD patterns of the products and thus automatically determine whether the experiments were successful. Decision tree analysis was used to visualize the experimental results, after extracting factors that mainly affected the synthetic reproducibility. Water adsorption isotherms revealed that KGF-3 possesses unique hydrophilic pores. Impedance measurements demonstrated good proton conductivities $(\sigma=5.2 \times$ $10^{-4} \mathrm{~S} \mathrm{~cm}^{-1}$ for KGF-3(Y)) at a high temperature (363 K) and relative humidity of $95 \% \mathrm{RH}$.
\end{abstract}

\section{Introduction}

Crystalline reticular materials, such as metal-organic frameworks (MOFs) and covalent organic frameworks (COFs), have been widely studied as promising environmental energy materials. ${ }^{[1]}$ Crystallization of these materials has been mainly achieved by solvothermal or hydrothermal synthesis in sealed reaction vessels. ${ }^{[1,2, ~ 2] ~ U n f o r t u n a t e l y, ~ t h e ~ d i s c o v e r y ~ o f ~ n e w ~ m a t e r i a l s ~ b y ~}$ such synthetic methods often requires patient trial and error rather than precise reaction design. Hence, it is desirable to understand the crystallization mechanism of reticular materials under solvothermal conditions, and develop a rational approach for selective crystallization of target compounds. ${ }^{[3]}$ Although many in situ measurements have been conducted to understand the crystallization process in a sealed reaction vessel, identifying the relationship between the reaction parameters and the outcome of a reaction remains a challenge. Therefore, facile and reliable techniques to understand and control the target reaction, which can be applicable during the screening of a synthetic process, are necessary to efficiently discover novel crystalline reticular materials.

Machine learning has recently attracted attention as an efficient exploration tool, especially in the area of materials synthesis. ${ }^{[4]}$ In particular, several attempts of determining the crystallization conditions of materials using machine learning have been reported. It is beginning to be regarded as a powerful method. ${ }^{[5]}$ While the application of machine learning techniques to predict crystallization conditions for nanoporous materials seems promising, its application in the exploration of novel materials remains limited. To the best of our knowledge, no studies thus far have introduced machine learning as a tool for preparing unknown MOFs. This is partially due to the lack of training data; open databases for the exploration of unknown MOFs are limited and the generation of such training data is expensive. ${ }^{[5 g]}$ In addition, during the exploration of novel MOFs by screening syntheses, the compounds obtained in failure experiments are generally present as complex mixtures. Therefore, facile techniques to characterize such crude mixtures in a format suitable for analysis by machine learning is necessary.

In this work, we focus on lanthanide-based MOFs (Ln-MOFs), in which lanthanide ions or clusters are linked by organic linkers. LnMOFs are promising materials for a wide range of applications, such as proton conductors, and various porous, luminescent and magnetic materials. ${ }^{[6]}$ Furthermore, lanthanides have been previously reported to form giant clusters under solvothermal synthetic conditions. ${ }^{[7]}$ Thus, multiple or novel properties are expected owing to the synergism when such large clusters or more highly dimensional infinite structures are incorporated as secondary building units (SBUs) in the skeletons of MOFs. ${ }^{[1 a, 1 c, 8]}$ However, since the formation of lanthanide clusters is easily affected by the reaction conditions, rational design and syntheses of MOFs containing Ln-cluster-based giant or infinite SBUs 
remain a challenge. In addition, Ln-MOFs are known to exhibit crystal polymorphism owing to their flexible coordination nature. As a result, it is difficult to selectively synthesize crystal polymorphs, and the preparation of Ln-MOFs frequently suffers from poor reproducibility. Hence, a facile and intuitive means to evaluate the factors that predominantly influence reaction outcomes is needed.

Herein, we report the synthesis of novel Ln-MOF containing lanthanide-double-layer-based SBUs (KGF-3) assisted by machine learning. To the best of our knowledge, this is the first example of applying machine learning to solvothermal synthesis for the exploration of unknown MOFs. The synthetic results are evaluated using both cluster analysis and decision tree analysis. These analyses enabled us to determine the optimal conditions for the reproducible synthesis of KGF-3. Figure 1 shows the flow process used to optimize the synthetic conditions using machinelearning techniques. Water adsorption experiments and impedance measurements are also used to analyze the pores and the proton conductivity of the prepared KGF-3, respectively.

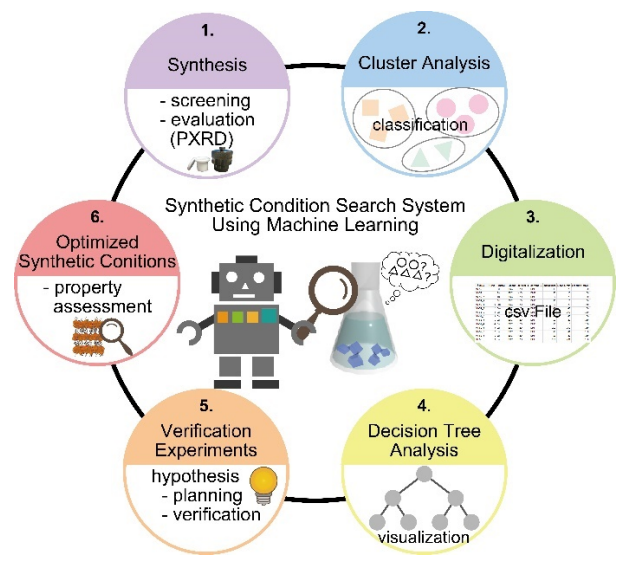

Figure 1. Schematic representation of the research flow process used to optimize the synthetic conditions by incorporating machine learning methods.

\section{Results and Discussion}

\section{Screening synthesis}

Solvothermal synthesis was performed using nitrate hexahydrate salts of lanthanide metal ions $\left(\mathrm{Ln}^{3+}=\mathrm{Sm}, \mathrm{Eu}, \mathrm{Gd}\right.$, and $\left.\mathrm{Tb}\right)$ in the presence of terephthalic acid $\left(\mathrm{H}_{2} \mathrm{BDC}\right)$ in $\mathrm{H}_{2} \mathrm{O} / \mathrm{DMF}$. A total of 108 experiments were conducted. Variables included the lanthanide metal ion, the concentration of the metal ion and/or ligand solution the reaction temperature and time, the cooling time, and the type of reaction vessel (see Synthetic Conditions 1 in the Experimental section). Solid powders were obtained under all conditions and were characterized by powder X-ray diffractometry (PXRD), where novel phases were observed in some cases.

The novel $\left[\mathrm{Ln}_{10}(\mathrm{BDC})_{3}(\mathrm{HCOO})_{4}\left(\mu_{3}-\mathrm{OH}\right)_{12}\left(\mu_{5}-\mathrm{CO}_{3}\right)_{4}\left(\mathrm{H}_{2} \mathrm{O}\right)_{2}\right]$ phase, which we refer to as "KGF-3", was obtained in the presence of various lanthanide ions. However, single crystals suitable for crystal structure analysis were obtained only when $\mathrm{Gd}^{3+}$ was used as the metal source. Based on the single-crystal X-ray data, KGF$3(\mathrm{Gd})$ was found to contain five types of non-equivalent $\mathrm{Gd}^{3+}$ ions with coordination numbers of eight or nine. Four complexes share ridges with each other to form a chain, which is then connected by another lanthanide ion to form a porous layer (Figure 2a). The two layers are cross-linked by carbonate ions (the origin of the carbonates will be discussed later) to form a double layer, while $\mathrm{BDC}$ bridges the layers as pillar molecules. This results in a threedimensional pillar-layered structure (Figures $2 \mathrm{~b}-2 \mathrm{c}$ ). The $\mu_{5^{-}}$ $\mathrm{CO}_{3}{ }^{2-}$ coordination mode is common in giant cluster synthesis, but is unusual in MOFs (Figure 2d). Moreover, we noted that formic acid generated by the decomposition of DMF was coordinated. Many $\mu$-OH groups are aligned on the KGF-3 pore surfaces and disordered guest molecules (most likely water) are incorporated in the pores, suggesting that the pores are highly hydrophilic in nature.

\section{Analysis by machine learning techniques}

KGF-3 was difficult to isolate, and its preparation suffered from poor reproducibility with different phases frequently being obtained under the same synthetic conditions; a pure KGF-3 phase was not obtained even after 108 experimental trials. To optimize the synthetic conditions, we extracted the dominant factors of the reaction using machine-learning techniques. To predict the conditions under which KGF-3 can be obtained more consistently, it was necessary to determine whether or not the reaction was successful using PXRD. However, the products are complex mixtures of different phases in many cases; hence, assigning each PXRD pattern to an appropriate phase is challenging. A simple method that excluded arbitrariness was required; therefore, we classified the obtained patterns using cluster analysis. ${ }^{[9]}$ All diffraction patterns were automatically analyzed and successfully classified into six categories, in which the main products were KGF-3 (cluster 1), four reported phases (clusters $2,{ }^{[10]} 3,{ }^{[11]}, 4,{ }^{[12]}$ and $5^{[13]}$ ), and an unknown phase (cluster 6) (Figures 3 and S1). These results revealed that the automated classification was consistent with the "researcher intuition", except two data points in the 108 patterns (see the Cluster Analysis section in the $\mathrm{SI}$ ).
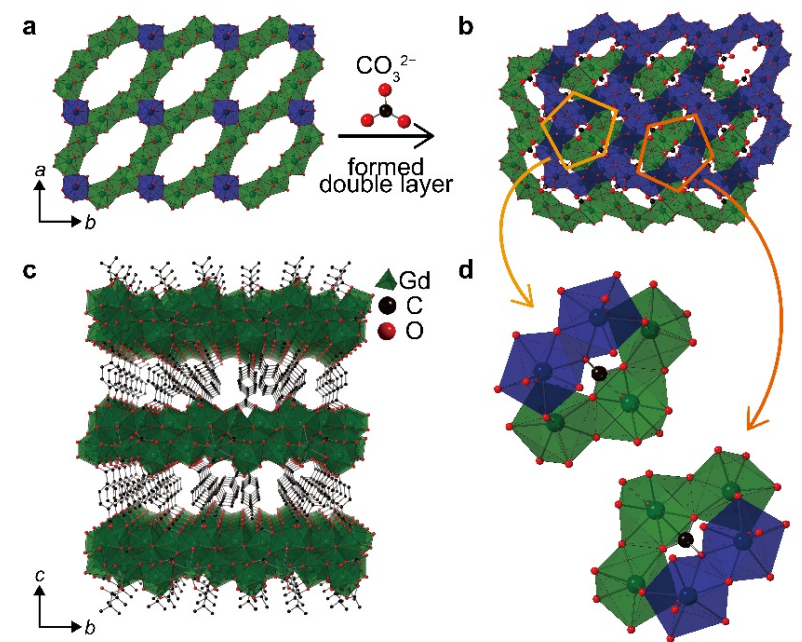

Figure 2. Crystal structure of KGF-3(Gd). (a) Monolayer with 10 spread-out lanthanide clusters. (b) Double layer connected by carbonate ions. (c) View along the a-axis. (d) Pentagonal pockets showing the connections imparted by the carbonate ions. $\mathrm{H}$ atoms are omitted for clarity. 


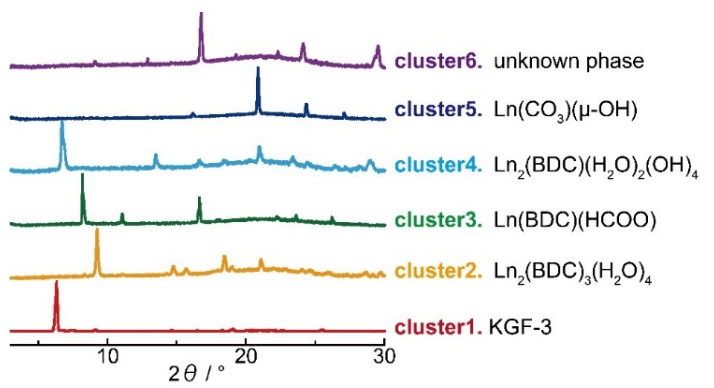

Figure 3. Using cluster analysis to classify the acquired PXRD patterns.

Next, we focused on extracting the dominant factors responsible for the poor reproducibility by employing decision tree analysis, which is considered to be one of the most interpretable machinelearning techniques. ${ }^{[5 a, 5 f, 5 h]}$ Initially, the experimental data and cluster analysis results were linked together in a text file, after which the data file was analyzed using the decision tree technique. Here, the objective variables were the crystal phases assigned by cluster analysis of the PXRD patterns, and the explanatory variables were the synthetic parameters (see the Decision Tree Analysis section in the SI). The results presented in Figure $4 \mathrm{a}$ suggest that the most suitable synthetic conditions for preparing KGF-3 are as follows: Ligand solution concentration, 18-22 mM; cooling time, $>12 \mathrm{~h}$; and metal salt source, company $\mathrm{A}$. The parameters appearing at the branches in the decision tree were also identified from a random forest analysis (Figure $4 b-4 c$ ). Thus, visualizing the experimental data by decision tree analysis allowed us to understand the synthetic conditions at a glance. The information extracted from the decision tree is summarized as follows. First, it is likely that when the ligand concentration is $<18$ $\mathrm{mM}$, a product is formed with bridging carbonate ligands (cluster 5) (Figure 4a-(1)), suggesting that carbonate is generated by the decomposition of DMF or through capture from the air. ${ }^{[14]}$ Secondly, the success or failure of the KGF-3 synthesis was determined by the reagent company employed, with the nitrate provided by company A being most suitable (Figure 4a-(2)). Finally, as shown in Figure $4 a-(3)$, the success rate was $17 \%$ on the left branch (Eu) and $45 \%$ on the right branch ( $\mathrm{Gd}$ and $\mathrm{Tb}$ ), suggesting that the metal ion affects the synthetic process. To determine the synthetic conditions that maximize reproducibility, the extracted dominant factors were verified as follows.

\section{Exploration of the optimized synthetic conditions}

In terms of the supply company for the lanthanide nitrate salt, the reagents purchased from company A were superior (Figure S3), exhibiting higher purity (99.95\%) compared to those obtained from companies B (99.9\%) and C (99.5\%). The purities guaranteed by the reagent companies were evaluated based only on the metal ion concentration. To estimate the influence of the purity of the metal source, $\mathrm{Tb}\left(\mathrm{NO}_{3}\right)_{3} \cdot 6 \mathrm{H}_{2} \mathrm{O}$ with the highest purity $(99.999 \%)$, i.e., from company D, was also used. This resulted in
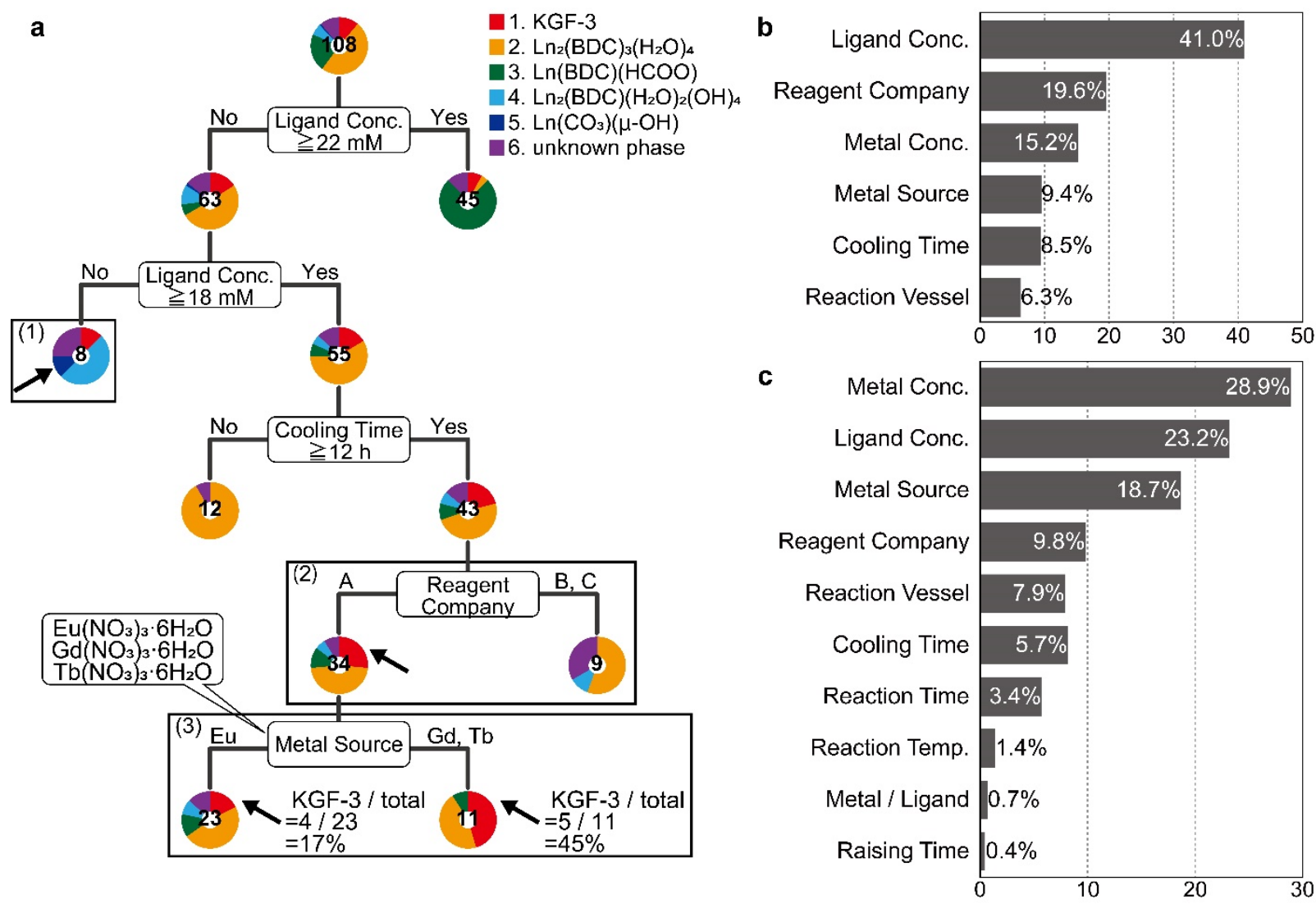

Figure 4. (a) Visualization of the relationships between the experimental conditions and the products based on decision tree analysis. Ovals represent decision nodes, the pie charts show the product ratios, and the number of experiments is shown in the center of each pie chart; (1), (2), and (3) are branches related to the concentration conditions, the reagent company, and the lanthanide metal, respectively. Branches that are not discussed in the main text have been omitted. The complete version of the decision tree is given in Figure S2. The percentage of each parameter that contributes to the branch is shown. (b) Decision tree analysis. (c) Random forest analysis. 


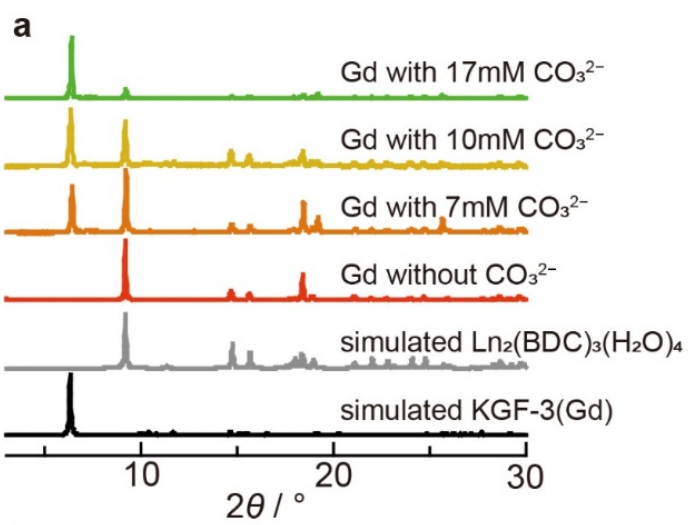

b

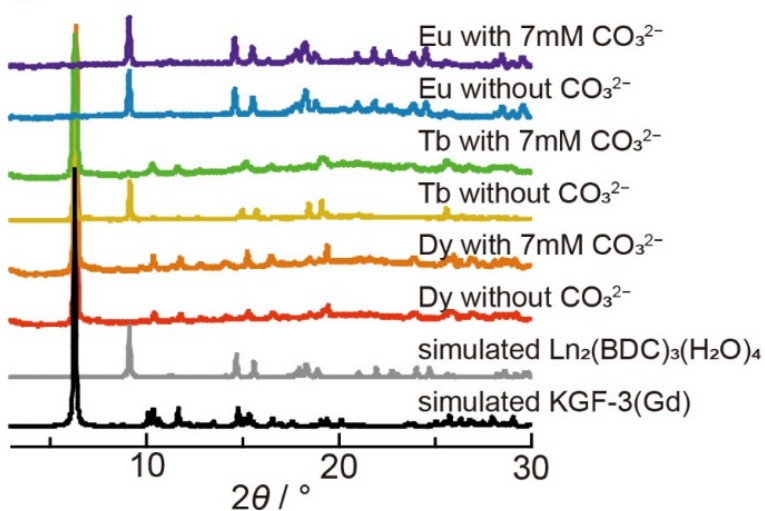

Figure 5. PXRD patterns obtained in the presence of sodium carbonate. (a) After the addition of various concentrations of sodium carbonate to Gd. (b) Comparisons with and without the addition of sodium carbonate for Eu, Tb, and Dy.

an improved success rate compared to that obtained using $\mathrm{Tb}\left(\mathrm{NO}_{3}\right)_{3} \cdot 6 \mathrm{H}$ O from company $\mathrm{A}$; the success rate was $20 \%$ (7 out of 34 trials) using the $\mathrm{Tb}$ source from company $\mathrm{A}$, while the success rate increased to $95 \%$ using the Tb source from company D (19 out of 20 trials) (Figure S4). The different purities were evaluated by inductively coupled plasma-mass spectrometry, which revealed that the $\mathrm{Tb}\left(\mathrm{NO}_{3}\right)_{3} \cdot 6 \mathrm{H}_{2} \mathrm{O}$ obtained from company A contained slightly higher levels of Eu than that from company $D$ (Table S2). Therefore, KGF-3 was prepared using lanthanide salts purchased from company $D$ in all of the following experiments.

The results from the decision tree analysis also showed that under low concentration conditions, carbonate ions are captured within the structure (cluster 5). Generally, carbonate ions play important roles as anion templates during the formation of polynuclear lanthanide clusters. ${ }^{[7 e, 15]}$ In many cases, carbonate ions are generated by the decomposition of the precursor and/or uptake of carbon dioxide from the air. Therefore, in the crystal structure of KGF-3, it is likely that the molecules coordinating to five metal ions in a pentagonal pocket (Figure $2 \mathrm{~d}$ ) are carbonate ions. To estimate the effect of these carbonate ions, KGF-3 was synthesized by adding sodium carbonate (Synthetic Conditions 2). For $\mathrm{Gd}$ and $\mathrm{Tb}$, the formation of the $\mathrm{Ln}_{2}(\mathrm{BDC})_{3}\left(\mathrm{H}_{2} \mathrm{O}\right)_{4}$ impurity (cluster 2) was suppressed with the addition of the carbonate ions. In the case of Eu, $\operatorname{Ln}_{2}(B D C)_{3}\left(\mathrm{H}_{2} \mathrm{O}\right)_{4}$ (cluster 2) was preferentially synthesized, regardless of whether carbonate ions were added or not (Figure 5). These results suggest that the effect imparted by the carbonate ions depends on the lanthanide metal ion.
The decision tree analysis (Figure 4a-(3)) and the response to the addition of carbonate ions strongly suggest that the metal ion affects the probability of successfully synthesizing KGF-3. Therefore, we synthesized KGF-3(Ln) with various lanthanide metal ions ( $\mathrm{La}-\mathrm{Lu}$, excluding $\mathrm{Pm}$ ) under optimized Synthetic Conditions 3, 4, 5, and 6 (Figures 6 and S5). The PXRD and elemental analysis (EA) revealed that only Dy and Ho provided pure isolated KGF-3, and only the EA data for Dy (ionic radius, $r$ $=1.03 \AA)$ and $\mathrm{Ho}(r=1.02 \AA)$ matched the calculated values. This suggests that the ionic radius is important for the selective synthesis of pure KGF-3, and the optimal ionic radius is 1.02-1.03 $\AA$. The EA results indicate that no nitrogen is present in KGF-3 (Table S4), thereby confirming that all three directional ligands in the crystal structure are carbonate ions (see the Elemental Analysis section in the SI). The observation that trace amounts of Eu present in the purchased reagent increase the chances of a failed experiment coincides with the fact that other crystal phases form with high probabilities using Eu. In many cases, the ionic radius of the lanthanide ion strongly influences the construction of the obtained Ln complex. ${ }^{[16]}$ In fact, KGF-3(Y) was successfully isolated when $\mathrm{Y}^{3+}(r=1.02 \AA)$, whose ionic radius is close to those of $\mathrm{Dy}^{3+}$ and $\mathrm{Ho}^{3+}$ (Figure 6), was used. This strongly suggests that the ionic radius of the lanthanide metal is a crucial and dominant factor for KGF-3 formation.

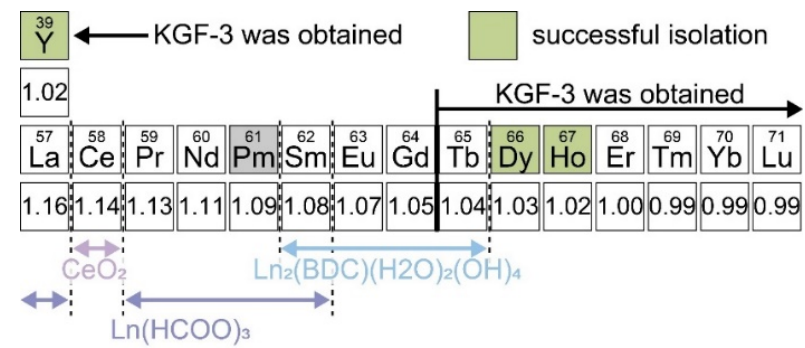

Figure 6. Summary of the synthetic results for KGF-3 under Synthetic Conditions 3 and 4 . See Figure S5 for details. KGF-3(Dy and Y) were successfully isolated under Synthetic Conditions 3 , while KGF-3(Ho) was successfully isolated under Synthetic Conditions 4.

\section{Adsorption properties and proton conductivity} measurements of KGF-3

To evaluate the permanent porosity of KGF-3(Dy, Ho, and $\mathrm{Y}$ ), $\mathrm{N}_{2}$ and $\mathrm{H}_{2} \mathrm{O}$ adsorption isotherms were acquired (Figures $7 \mathrm{a}$ and $\mathrm{S} 8$ ). The results suggest that KGF-3 adsorbs $\mathrm{H}_{2} \mathrm{O}$ molecules in its pores, whereas $N_{2}$ is not adsorbed; hence KGF-3 is likely to possess narrow hydrophilic channels with diameters that are too small for nitrogen diffusion at $77 \mathrm{~K}$. This result is consistent with the obtained crystal structure of KGF-3(Gd). Although water molecules are disordered within the pores, they are expected to form a pathway for proton conduction and the water assembly is stabilized by the hydrophilic pore surface (Figure S10). Therefore, the hydrophilic nature of KGF-3 prompted us to evaluate its proton conductivity. Accordingly, the alternating current impedances of KGF-3(Dy, Ho, and Y) were measured at 313-363 K and 95\% relative humidity (Figures $7 \mathrm{~b}$ and S11). KGF-3 retained its crystalline nature after the impedance experiments and even after soaking in water (Figures S9 and S11). Further, KGF-3(Y) showed the highest proton conductivity among the three MOFs (i.e., $5.2 \times 10^{-4} \mathrm{~S} \mathrm{~cm}^{-1}$ at $363 \mathrm{~K}$ ). The conductivity of KGF-3(Y) increased with increasing temperature as a result of thermal activation of the water molecules. The activation energy for proton 

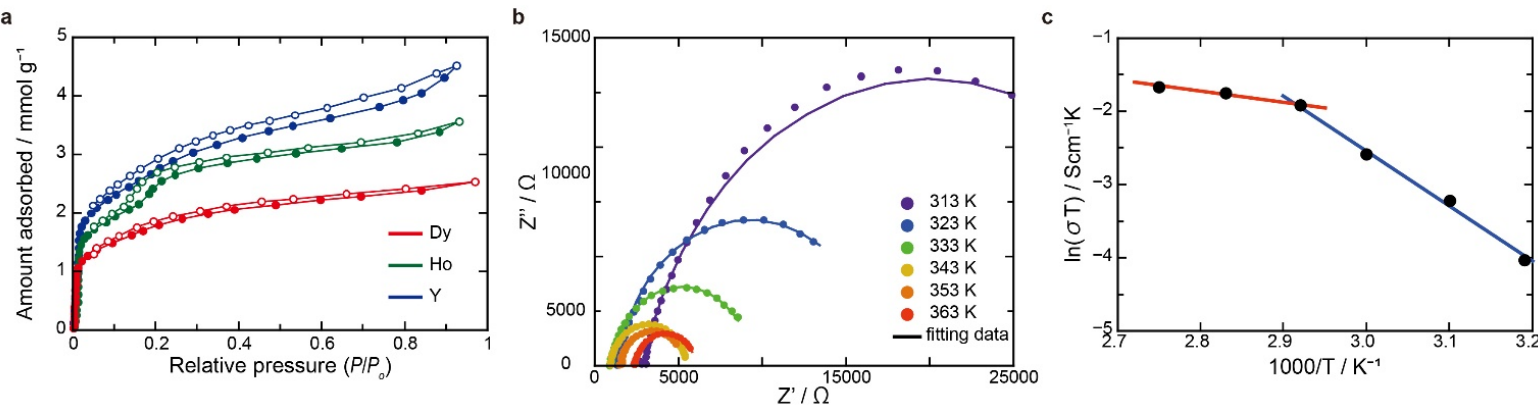

Figure 7. (a) Adsorption and desorption isotherms for $\mathrm{H}_{2} \mathrm{O}$. The solid and open symbols correspond to adsorption and desorption, respectively. (b) Nyquist plots at various temperatures and at $95 \% \mathrm{RH}$ for a pellet sample of KGF-3(Y). (c) Arrhenius plot of the ion conductivity at $95 \%$ RH of KGF-3(Y).

conduction was calculated to be $0.65 \mathrm{eV}$ at low temperatures (40$343 \mathrm{~K})$, whereas it was $0.14 \mathrm{eV}$ at high temperatures (343-363 K) (Figure 7c). This suggests that a change in the transport mechanism occurs at $\sim 343 \mathrm{~K} \cdot{ }^{[17]}$

\section{Conclusion}

In summary, we successfully synthesized a series of novel pillarlayered Ln-MOFs containing lanthanide double-layer-based SBUs, referred to as "KGF-3." Although it was difficult to isolate KGF-3 in our initial screening experiments owing to poor synthetic reproducibility, we successfully extracted the dominant factors for KGF-3 synthesis by evaluating both failure and success using machine-learning techniques. Our results indicate that the lanthanide ion affects the synthesis results. Further, systematic synthetic experiments demonstrated the effect of the ionic radius of the metal ion. This method is a useful tool for preparing new MOFs and related compounds, such as coordination polymers and COFs that suffer from poor synthetic reproducibility. We are currently applying this method to the exploration of novel MOFs and coordination polymers, for which obtaining highly crystalline samples is challenging. The results will be presented in due course.

\section{Experimental Section}

\section{General characterization and analytical methods}

See the Supplementary Information for further details.

\section{Synthetic Conditions 1 (Exploratory experiments)}

$\mathrm{Ln}\left(\mathrm{NO}_{3}\right)_{3} \cdot 6 \mathrm{H}_{2} \mathrm{O}(\mathrm{Ln}=\mathrm{Sm}, \mathrm{Eu}, \mathrm{Gd}, \mathrm{Tb}, 0.008-0.8 \mathrm{mmol})$ was dissolved in distilled water (2-200 $\mathrm{mM})$, and 2-hydroxyterephthalic acid $\left(\mathrm{H}_{2} \mathrm{BDC}\right.$ $0.008-0.8 \mathrm{mmol}$ ) was separately dissolved in $\mathrm{N}, \mathrm{N}$-dimethylformamide (DMF; 2-200 mM). These two solutions were mixed in a Teflon-lined stainless-steel container $(4,8,16,30$, or $100 \mathrm{~mL})$ and heated at 145 or $150{ }^{\circ} \mathrm{C}$ for $24-80 \mathrm{~h}$. At the end of the heating process, the container was cooled to $30^{\circ} \mathrm{C}$. The heating time was either 5 or $12 \mathrm{~h}$, and the cooling time was either rapid (10 $\mathrm{min})$ or slow $(12-80 \mathrm{~h})$.

\section{Synthetic Conditions 2 (Carbonate ion-added synthesis)}

Three separate solutions were prepared: $\mathrm{Ln}\left(\mathrm{NO}_{3}\right)_{3} \cdot 6 \mathrm{H}_{2} \mathrm{O}(\mathrm{Ln}=\mathrm{Eu}, \mathrm{Gd}, \mathrm{Tb})$ was dissolved in distilled water $(0.08 \mathrm{mmol}, 2.4 \mathrm{~mL}), \mathrm{H}_{2} \mathrm{BDC}$ was dissolved in DMF $(0.08 \mathrm{mmol}, 1.6 \mathrm{~mL})$, and $\mathrm{Na}_{2} \mathrm{CO}_{3}$ was dissolved in distilled water
$(0-0.036 \mathrm{mmol}, 2.0 \mathrm{~mL})$. These three solutions were then mixed in an 8 $\mathrm{mL}$ Teflon-lined stainless-steel container and heated at $150^{\circ} \mathrm{C}$ for $48 \mathrm{~h}$. At the end of the heating process, the container was cooled to $30^{\circ} \mathrm{C}$. The heating time was $5 \mathrm{~h}$, and the cooling time was $12 \mathrm{~h}$.

Synthetic Conditions 3 (Optimized Synthetic Conditions A: Stoichiometric synthesis)

$\mathrm{Ln}\left(\mathrm{NO}_{3}\right)_{3} \cdot \mathrm{xH}_{2} \mathrm{O}(\mathrm{Ln}=\mathrm{La}, \mathrm{Ce}, \mathrm{Pr}, \mathrm{Nd}, \mathrm{Sm}, \mathrm{Eu}, \mathrm{Gd}, \mathrm{Tb}, \mathrm{Dy}, \mathrm{Ho}, \mathrm{Er}, \mathrm{Tm}, \mathrm{Yb}$, $\mathrm{Lu}$, or $\mathrm{Y})$ was dissolved in distilled water $(0.16 \mathrm{mmol}, 4.8 \mathrm{~mL})$ and $\mathrm{H}_{2} \mathrm{BDC}$ was separately dissolved in DMF $(0.048 \mathrm{mmol}, 3.2 \mathrm{~mL})$. These two solutions were mixed in a $16 \mathrm{~mL}$ Teflon-lined stainless-steel container and heated at $150^{\circ} \mathrm{C}$ for $48 \mathrm{~h}$. At the end of the heating process, the container was cooled to $30^{\circ} \mathrm{C}$. The heating time was $5 \mathrm{~h}$, and the cooling time was $12 \mathrm{~h}$. The obtained residue was washed with DMF and $\mathrm{MeOH}(\times 3$ for each solvent).

Synthetic Conditions 4 (Optimized Synthetic Conditions B: Preparation using excess $\mathrm{H}_{2} \mathrm{BDC}$ )

$\mathrm{Ln}\left(\mathrm{NO}_{3}\right)_{3} \cdot \mathrm{xH}_{2} \mathrm{O}(\mathrm{Ln}=\mathrm{La}, \mathrm{Ce}, \mathrm{Pr}, \mathrm{Nd}, \mathrm{Sm}, \mathrm{Eu}, \mathrm{Gd}, \mathrm{Tb}, \mathrm{Dy}, \mathrm{Ho}, \mathrm{Er}, \mathrm{Tm}, \mathrm{Yb}$ $\mathrm{Lu}$, or $\mathrm{Y})$ was dissolved in distilled water $(0.16 \mathrm{mmol}, 4.8 \mathrm{~mL})$ and $\mathrm{H}_{2} \mathrm{BDC}$ was separately dissolved in DMF $(0.16 \mathrm{mmol}, 3.2 \mathrm{~mL})$. These two solutions were mixed and separated by centrifugation, and the supernatant was placed in a $16 \mathrm{~mL}$ Teflon-lined stainless-steel container and heated at $150{ }^{\circ} \mathrm{C}$ for $48 \mathrm{~h}$. At the end of the heating process, the container was cooled to $30^{\circ} \mathrm{C}$. The heating time was $5 \mathrm{~h}$, and the cooling time was $12 \mathrm{~h}$. The obtained residue was washed with $\mathrm{DMF}$ and $\mathrm{MeOH}(\times 3$ for each solvent).

Synthetic Conditions 5 (Optimized synthetic conditions for KGF-3(Eu, Gd))

Three separate solutions were prepared: $\operatorname{Ln}\left(\mathrm{NO}_{3}\right)_{3} \cdot 6 \mathrm{H}_{2} \mathrm{O}(\mathrm{Ln}=\mathrm{Eu}, \mathrm{Gd})$ was dissolved in distilled water $(0.08 \mathrm{mmol}, 2.4 \mathrm{~mL}), \mathrm{H}_{2} \mathrm{BDC}$ was dissolved in DMF $(0.024 \mathrm{mmol}, 1.6 \mathrm{~mL})$, and $\mathrm{Na}_{2} \mathrm{CO} 3$ was dissolved in distilled water $(0.04 \mathrm{mmol}, 2.0 \mathrm{~mL})$. These three solutions were then mixed in an $8 \mathrm{~mL}$ Teflon-lined stainless-steel container and heated at $150{ }^{\circ} \mathrm{C}$ for $6 \mathrm{~h}$. At the end of the heating process, the container was cooled to $30^{\circ} \mathrm{C}$. The heating time was $5 \mathrm{~h}$, and the cooling time was $12 \mathrm{~h}$. The residue was washed with DMF and $\mathrm{MeOH}$ ( $\times 3$ for each solvent).

Synthetic Conditions 6 (Optimized synthetic conditions for KGF3(Tb))

$\mathrm{Tb}\left(\mathrm{NO}_{3}\right)_{3} \cdot 6 \mathrm{H}_{2} \mathrm{O}$ was dissolved in distilled water $(0.08 \mathrm{mmol}, 2.4 \mathrm{~mL})$ and $\mathrm{H}_{2} \mathrm{BDC}$ was separately dissolved in DMF $(0.024 \mathrm{mmol}, 1.6 \mathrm{~mL})$. These two solutions were mixed in an $8 \mathrm{~mL}$ Teflon-lined stainless-steel container and heated at $150^{\circ} \mathrm{C}$ for $48 \mathrm{~h}$. At the end of the heating process, the container was cooled to $30^{\circ} \mathrm{C}$. The heating time was $5 \mathrm{~h}$, and the cooling time was 
$12 \mathrm{~h}$. The obtained residue was washed with $\mathrm{DMF}$ and $\mathrm{MeOH}(\times 3$ for each solvent).

\section{Preparation of a single crystal of KGF-3(Gd)}

Three solutions were prepared separately: $\mathrm{Gd}\left(\mathrm{NO}_{3}\right)_{3} \cdot 6 \mathrm{H}_{2} \mathrm{O}$ was dissolved in distilled water $(0.08 \mathrm{mmol}, 2.4 \mathrm{~mL}), \mathrm{H}_{2} \mathrm{BDC}$ was dissolved in DMF $(0.024$ $\mathrm{mmol}, 1.6 \mathrm{~mL})$, and $\mathrm{Na}_{2} \mathrm{CO}_{3}$ was dissolved in distilled water $(0.04 \mathrm{mmol}$, $2.0 \mathrm{~mL}$ ). These three solutions were then mixed in an $8 \mathrm{~mL}$ Teflon-lined stainless-steel container and heated at $150{ }^{\circ} \mathrm{C}$ for $6 \mathrm{~h}$. At the end of the heating process, the container was cooled to $30^{\circ} \mathrm{C}$. The heating time was $5 \mathrm{~h}$, and the cooling time was $12 \mathrm{~h}$.

\section{Machine-learning analysis}

Cluster analysis was carried out using PDXL 2.8 (Rigaku). Decision tree analysis (Partition) was performed using JMP $®$ Pro14.3, and random forest analysis (Bootstrap Forest) was carried out using JMP® Pro 15.2.1 (SAS Institute Inc., Cary, NC, USA).

\section{Acknowledgements}

This work was supported by grants from JST PRESTO (Grant No. JPMJPR17NA) and JSPS KAKENHI (Grant Nos. 20H02577, 17K00320, 20H04680, and 20H04646). We thank Dr. Satoki Okabayashi, Prof. Koichi Chiba, and Dr. Akane Ito (Kwansei Gakuin University) for ICP-OES support. This work was performed under the Cooperative Research Program of "Network Joint Research Center for Materials and Devices." Prof. Masaharu Tanimizu was supported by a Joint Research Grant for the ICP-MS experiments from the Environmental Isotope Study of the Research Institute for Humanity and Nature.

Keywords: lanthanides • metal-organic frameworks • machine learning $\bullet$ solvothermal synthesis $\bullet$ proton conductivity

[1] a) O. M. Yaghi, M. O'Keeffe, N. W. Ockwig, H. K. Chae, M. Eddaoudi, J. Kim, Nature 2003, 423, 705-714; b) S. Kitagawa, R. Kitaura, S. Noro, Angew. Chem. Int. Ed. 2004, 43, 2334-2375; c) G. Ferey, Chem. Soc. Rev. 2008, 37, 191-214; d) S. Das, P. Heasman, T. Ben, S. Qiu, Chem. Rev. 2017, 117, 1515-1563; e) K. V. Kumar, K. Preuss, M. M. Titirici, F. Rodriguez-Reinoso, Chem. Rev. 2017, 117, 1796-1825.

[2] M. Kumar, L.-Q. Li, J. K. Zaręba, L. Tashi, S. C. Sahoo, M. Nyk, S.-J. Liu, H. N. Sheikh, Cryst. Growth Des. 2020, 20, 6430-6452.

[3] a) M. G. Goesten, M. F. de Lange, A. I. Olivos-Suarez, A. V. Bavykina, P. Serra-Crespo, C. Krywka, F. M. Bickelhaupt, F. Kapteijn, J. Gascon, Nat. Commun. 2016, 7, 11832; b) S. Saha, S. Springer, M. E. Schweinefuß, D. Pontoni, M. Wiebcke, K. Huber, Cryst. Growth Des. 2016 , 16, 2002-2010; c) H. H. Yeung, Y. Wu, S. Henke, A. K. Cheetham, D. O'Hare, R. I. Walton, Angew. Chem. Int. Ed. 2016, 55, 2012-2016; d) M. J. Van Vleet, T. Weng, X. Li, J. R. Schmidt, Chem. Rev. 2018, 118, 3681-3721.

[4] a) X. Yang, Y. Wang, R. Byrne, G. Schneider, S. Yang, Chem. Rev. 2019, 119, 10520-10594; b) C. W. Coley, N.
S. Eyke, K. F. Jensen, Angew. Chem. Int. Ed. 2020, 59, 22858-22893; c) S. M. Moosavi, K. M. Jablonka, B. Smit, J. Am. Chem. Soc. 2020, 142, 20273-20287.

[5] a) P. Raccuglia, K. C. Elbert, P. D. Adler, C. Falk, M. B. Wenny, A. Mollo, M. Zeller, S. A. Friedler, J. Schrier, A. J. Norquist, Nature 2016, 533, 73-76; b) V. Duros, J. Grizou, W. Xuan, Z. Hosni, D. L. Long, H. N. Miras, L. Cronin, Angew. Chem. Int. Ed. 2017, 56, 10815-10820; c) R. L. Greenaway, V. Santolini, M. J. Bennison, B. M. Alston, C. J. Pugh, M. A. Little, M. Miklitz, E. G. B. Eden-Rump, R. Clowes, A. Shakil, H. J. Cuthbertson, H. Armstrong, M. E. Briggs, K. E. Jelfs, A. I. Cooper, Nat. Commun. 2018, 9, 2849; d) V. Duros, J. Grizou, A. Sharma, S. H. M. Mehr, A. Bubliauskas, P. Frei, H. N. Miras, L. Cronin, J. Chem. Inf. Model. 2019, 59, 2664-2671; e) S. M. Moosavi, A. Chidambaram, L. Talirz, M. Haranczyk, K. C. Stylianou, B. Smit, Nat. Commun. 2019, 10, 539; f) K. Muraoka, Y. Sada, D. Miyazaki, W. Chaikittisilp, T. Okubo, Nat. Commun. 2019, 10, 4459; g) K. M. Jablonka, D. Ongari, S. M. Moosavi, B. Smit, Chem. Rev. 2020, 120, 8066-8129; h) Y. Xie, C. Zhang, X. Hu, C. Zhang, S. P. Kelley, J. L. Atwood, J. Lin, J. Am. Chem. Soc. 2020, 142, 1475-1481.

[6] a) J. Rocha, L. D. Carlos, F. A. Paz, D. Ananias, Chem. Soc. Rev. 2011, 40, 926-940; b) S. Roy, A. Chakraborty, T. K. Maji, Coord. Chem. Rev. 2014, 273, 139-164; c) W. P. Lustig, S. Mukherjee, N. D. Rudd, A. V. Desai, J. Li, S. K. Ghosh, Chem. Soc. Rev. 2017, 46, 3242-3285; d) X. Meng, H. N. Wang, S. Y. Song, H. J. Zhang, Chem. Soc. Rev. 2017, 46, 464-480; e) F. Saraci, V. Quezada-Novoa, P. R. Donnarumma, A. J. Howarth, Chem. Soc. Rev. 2020, 49, 7949-7977.

[7] a) J. B. Peng, X. J. Kong, Q. C. Zhang, M. Orendac, J. Prokleska, Y. P. Ren, L. S. Long, Z. Zheng, L. S. Zheng, J. Am. Chem. Soc. 2014, 136, 17938-17941; b) J. Dong, P. Cui, P. F. Shi, P. Cheng, B. Zhao, J. Am. Chem. Soc. 2015, 137, 15988-15991; c) K. Wang, Z. L. Chen, H. H. Zou, K. Hu, H. Y. Li, Z. Zhang, W. Y. Sun, F. P. Liang, Chem. Commun. 2016, 52, 8297-8300; d) J. Wang, M. Feng, M. N. Akhtar, M. L. Tong, Coord. Chem. Rev. 2019, 387, 129-153; e) X.-Y. Zheng, J. Xie, X.-J. Kong, L.-S. Long, L.-S. Zheng, Coord. Chem. Rev. 2019, 378, $222-$ 236.

[8] a) T. Devic, C. Serre, Chem. Soc. Rev. 2014, 43, 6097$6115 ;$ b) H. N. Miras, L. Vila-Nadal, L. Cronin, Chem. Soc. Rev. 2014, 43, 5679-5699.

[9] B. M. Butler, A. M. Sila, K. D. Shepherd, M. Nyambura, C. J. Gilmore, N. Kourkoumelis, S. Hillier, Geoderma 2019, 337, 413-424.

[10] T. M. Reineke, M. Eddaoudi, M. Fehr, D. Kelley, O. M. Yaghi, Journal of the American Chemical Society 1999, 121, 1651-1657.

[11] G. Huang, P. Yang, N. Wang, J.-Z. Wu, Y. Yu, Inorg. Chim. Acta 2012, 384, 333-339.

[12] C. Serre, F. Millange, J. Marrot, G. Ferey, Chem. Mater. 2002, 14, 2409-2415. 
[13] H. Tian, L. Zhao, Y. N. Guo, Y. Guo, J. Tang, Z. Liu, Chem. Commun. 2012, 48, 708-710.

[14] a) B. Zhang, X. Zheng, H. Su, Y. Zhu, C. Du, M. Song, Dalton Trans. 2013, 42, 8571-8574; b) H.-M. Chen, W.-M. Wang, X.-Q. Li, X.-Y. Chu, Y.-Y. Nie, Z. Liu, S.-X. Huang, H.-Y. Shen, J.-Z. Cui, H.-L. Gao, Inorg. Chem. Front. 2018, 5, 394-402.

[15] a) X. Y. Zheng, Y. H. Jiang, G. L. Zhuang, D. P. Liu, H. G. Liao, X. J. Kong, L. S. Long, L. S. Zheng, J. Am. Chem. Soc. 2017, 139, 18178-18181; b) Y. Zhou, X. Y. Zheng, J. Cai, Z. F. Hong, Z. H. Yan, X. J. Kong, Y. P. Ren, L. S. Long, L. S. Zheng, Inorg. Chem. 2017, 56, 2037-2041; c) X. Y. Li, H. F. Su, Q. W. Li, R. Feng, H. Y. Bai, H. Y. Chen, J. Xu, X. H. Bu, Angew. Chem. Int. Ed. 2019, 58, 1018410188.

[16] a) H. P. Xiao, J. Zhou, R. Q. Zhao, W. B. Zhang, Y. Huang, Dalton Trans. 2015, 44, 6032-6039; b) M. Ellart, F. Blanchard, M. Rivenet, F. Abraham, Inorg. Chem. 2020, 59, 491-504.

[17] a) P. Ramaswamy, N. E. Wong, G. K. Shimizu, Chem. Soc. Rev. 2014, 43, 5913-5932; b) D. W. Lim, H. Kitagawa, Chem. Rev. 2020, 120, 8416-8467. 


\section{Entry for the Table of Contents}

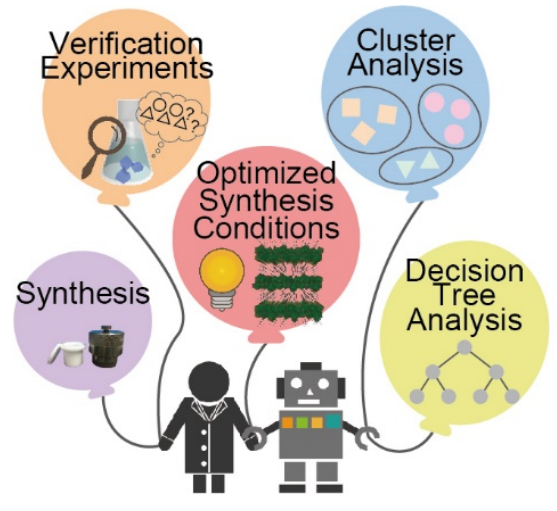

A series of novel metal organic frameworks with lanthanide double-layer-based inorganic subnetworks (KGF-3) was synthesized assisted by machine learning. The synthetic conditions were successfully optimized by extracting the dominant factors for KGF-3 synthesis using two machine-learning techniques. KGF-3 possesses unique hydrophilic pores, and impedance measurements demonstrated good proton conductivities.

Institute and/or researcher Twitter usernames: @DaisukeTanakaKG 